\title{
Amiodarone concentrations
} in plasma and fat tissue

\section{during chronic treatment and related toxicity}

\section{Carmelo Lafuente-Lafuente, ${ }^{1,2}$ Jean-Claude Alvarez, ${ }^{3,4}$}

Antoine Leenhardt, ${ }^{5,6}$ Stéphane Mouly, ${ }^{1,2}$ Fabrice Extramiana, ${ }^{5,6}$

Charles Caulin, ${ }^{1,2}$ Christian Funck-Brentano ${ }^{7,8,9} \&$

Jean-François Bergmann ${ }^{1,2}$

${ }^{1}$ AP-HP, Hôpital Lariboisière, Department of Internal Medicine A and ${ }^{2}$ Univ Paris 07, Department of

Therapeutics, Paris, ${ }^{3}$ AP-HP, Hôpital Raymond Poincaré, Laboratory of Pharmacology and Toxicology, Garches, ${ }^{4}$ Univ Paris-lle-de-France Ouest (PIFO), Department of Pharmacology, ${ }^{5}$ AP-HP, Hôpital Lariboisière, Department of Cardiology, ${ }^{6}$ Univ Paris 07, Department of Cardiology, ${ }^{7}$ AP-HP, Hôpital Saint-Antoine, Department of Pharmacology, ${ }^{8}$ UPMC Univ Paris 06, Department of Pharmacology, and 9 INSERM, CIC-9304, Paris, France

\section{Correspondence}

Dr Carmelo Lafuente-Lafuente, Clinique Thérapeutique - Médecine Interne A, Hôpital Lariboisière, Assistance Publique-Hôpitaux de Paris, 2 rue Ambroise Paré, 75010 Paris, France.

Tel: + $33149956339-9024$

Fax: + 33149956340

E-mail: c.lafuente@nodo3.net

\section{Study registration}

ClinicalTrials.gov ID: NCT00313443.

\section{Keywords}

amiodarone, amiodarone adverse events, fat tissue drug concentrations, plasma drug concentrations, tissue accumulation

Received

18 June 2008

Accepted

15 January 2009

\section{WHAT IT IS ALREADY KNOW ABOUT THIS SUBJECT}

- Amiodarone is a highly effective antiarrhythmic drug, but is limited in practice by its adverse effects, which are frequent on long-term administration and can be severe. - Amiodarone is very lipophilic and attains high concentrations in many tissues.

- Excessive accumulation in tissues is suspected as a possible cause of some of its adverse events, but sampling affected tissues (e.g. lung, thyroid, heart) in vivo is difficult. - Subcutaneous adipose tissue is more easily obtained, and studying concentrations of amiodarone and $\mathrm{N}$-desethyl-amiodarone there could help to understand how amiodarone distributes and accumulates in tissues in general.

\section{WHAT THIS STUDY ADDS}

- No evidence of excessive or unexpected accumulation of amiodarone in fat tissue, with respect to dose, was found in patients treated for $>3$ months.

- Concentrations in plasma and subcutaneous fat tissue were better related to maintenance daily dose than to cumulated dose or treatment duration.

- Clinically relevant adverse effects of amiodarone, for the most part hypothyroidism, were significantly associated with longer duration of treatment and larger cumulated doses.

- In contrast, adverse events were not correlated with higher amiodarone or $\mathrm{N}$-desethyl-amiodarone concentrations, whether in plasma or in adipose subcutaneous tissue. - The measurement of these concentrations does not seem useful in predicting the risk of adverse effects.

\section{AIMS}

To determine if amiodarone, highly lipophilic, accumulates in excess with respect to dose in fat tissue during long-term administration, and study if plasma and fat tissue concentrations are correlated with adverse effects.

\section{METHODS}

Trough concentrations of amiodarone and $\mathrm{N}$-desethyl-amiodarone were measured simultaneously in plasma and fat tissue, in 30 consecutive patients treated with amiodarone for 3 months to 12 years. Subcutaneous adipose tissue was obtained by needle aspiration from lumbar and abdominal areas. Concentrations were measured by liquid chromatography-tandem mass spectrometry.

\section{RESULTS}

Plasma levels of amiodarone and $\mathrm{N}$-desethyl-amiodarone were significantly correlated with daily maintenance doses $(R=0.52, P=0.003)$. Amiodarone concentrations in fat tissue were four to 226 times (mean 55) higher than in plasma, and well correlated with plasma levels $(R=0.68, P<0.001)$.

Concentrations of amiodarone and $\mathrm{N}$-desethyl-amiodarone in adipose tissue did not significantly increase with higher total cumulated doses or longer treatment duration. Nine of 12 patients who had received amiodarone for $\geq 2$ years developed clinically important adverse effects, predominantly hypothyroidism $(n=6)$, compared with two of 18 patients treated for less time (relative risk 6.75; $95 \%$ confidence interval 1.8,26). The incidence of those adverse effects was not significantly associated with amiodarone concentrations, whether in plasma or in adipose tissue.

\section{CONCLUSIONS}

We found no evidence of excessive or unexpected accumulation of amiodarone in fat tissue on long-term administration. Late amiodarone adverse effects, particularly hypothyroidism, are associated with longer exposure times, but do not seem to be explained by higher concentrations in plasma or in fat tissue. 


\section{Introduction}

Amiodarone is considered the most effective antiarrhythmic drug. Amiodarone has complex electrophysiological properties, combining effects from virtually all classes of antiarrhythmics with a predominant class III potassium channel blocker action, which makes it very effective in a variety of atrial and ventricular tachyarrhythmias [1]. A recent systematic review has shown that amiodarone is more effective than class I antiarrhythmics and sotalol in reducing recurrences of atrial fibrillation, the most frequent sustained arrhythmia [2].

However, clinical use of amiodarone is limited by its toxicity: adverse effects are frequent in long-term treatment. Even at low maintenance doses $-<400 \mathrm{mg}$ daily adverse effects lead to discontinuation of therapy in an average $23 \%$ of patients at 1 year [3] and as much as $41 \%$ in prolonged treatment [4]. Amiodarone side-effects may affect numerous organs: most frequently thyroid, skin and eyes, but also lung, liver, heart, and peripheral and central nervous system [3-6]. Some of these adverse effects can be severe or even fatal, especially lung toxicity (5-9\% of treated patients, $10 \%$ mortality [7]), but also uncontrolled hyperthyroidism, or rare cases of liver cirrhosis. The mechanism underlying amiodarone toxicity is not well known. High concentrations associated with lysosomal inclusion bodies have been observed in affected tissues [8]. However, different mechanisms are possible, depending on the affected organ: thyroid dysfunction is in part, although not solely, related to the high iodine content of amiodarone [9].

Amiodarone is very lipophilic and attains high concentrations in many tissues, especially in fat [8]. It is metabolized by the liver and eliminated in bile. Its main active metabolite is N-desethyl-amiodarone. Renal elimination is negligible $(<1 \%)$. As with any drug given at shorter intervals than its half-life, initial accumulation in tissues is expected following repeated administration, until steady state is reached. It has been suggested that amiodarone on long-term administration might be trapped in tissues and accumulate there in excess, beyond the expected amount, following a system which is not explained by classical pharmacokinetics and which would imply persistently increasing concentrations in plasma and tissues, with respect to maintenance dose [10]. That would explain the large variability between different studies in the estimation of basic pharmacokinetic parameters, as for example terminal half-life and steady-state volume of distribution $[11,12]$. Furthermore, such accumulation could explain why some of the amiodarone side-effects, particularly cutaneous and thyroid, increase in frequency over time [13].

To date, few studies have measured amiodarone concentration in vivo in human tissues and assessed its correlation with the cumulated dose or total treatment duration. They involved few patients and their results have been disparate [14-17]. It is difficult and risky to obtain in vivo samples of most tissues affected by amiodarone sideeffects (thyroid, lung, liver, heart, nerves) for analysing their amiodarone concentrations. As an alternative, subcutaneous adipose tissue can be easily obtained by needle aspiration and could be used to test if any unexpected tissue accumulation occurs. In such cases, amiodarone concentrations in fat tissue might be better related to the development of its late adverse effects than plasma concentrations.

We decided to study if amiodarone accumulated in excess in fat tissue on long-term administration, and to explore if fat tissue concentrations were more predictive of the development of adverse effects than plasma levels. To address these issues, we measured amiodarone trough concentrations simultaneously in plasma and in adipose tissue in 30 consecutive patients who were on chronic treatment with low-dose amiodarone for diverse periods of time. Our main objective was to determine if concentrations of amiodarone and $\mathrm{N}$-desethyl-amiodarone in adipose tissue were better correlated with the cumulated dose and total time of treatment than with the maintenance dose. A secondary objective was to explore the correlation of plasma and adipose tissue concentrations with the presence of adverse effects of amiodarone.

\section{Methods}

\section{Study design}

A series of consecutive patients on chronic treatment with amiodarone, in whom all samples - blood and two different needle aspirations of adipose subcutaneous tissue were obtained simultaneously, each patient one single time, to measure amiodarone concentrations.

\section{Patients}

Candidates for inclusion were any adult patient (age $>18$ years) attending the Internal Medicine or Cardiology Departments of our university hospital, who had been taking amiodarone for $>3$ months, independently of the indication and dose, on condition that treatment duration and cumulative dose could be accurately estimated. Exclusion criteria were: (i) local cutaneous lesions, infection or conditions that precluded sampling punctures; (ii) haemorrhagic risk conditions; and (iii) inability to give informed consent. Patients treated with antiplatelet drugs or prophylactic heparins were included, as well as patients on warfarin if the International Normalized Ratio was $<3.0$. The study was approved by the local human research review board (Comité de Protection des Personnes de Paris SaintLouis), and all patients gave written informed consent.

\section{Adipose tissue sampling}

Subcutaneous adipose tissue was obtained by needle aspiration following the technique of Beynen and Katan [18]: 
a skinfold is gripped with one hand and a 18-G needle inserted at $45^{\circ}$, then connected to vacuum using a $7-\mathrm{ml}$ evacuated tube (BD Vacutainer Systems, Plymouth, UK) and gently pushed back and forth a few times in the adipose layer to obtain a few fragments of tissue. To test if concentrations varied depending on the body region, samples were obtained at two different points: the abdominal wall and low lumbar region. All samplings - blood and adipose tissue - were performed simultaneously, just before each patient's daily administration of amiodarone (usually in the morning). Plasma and fat tissue were immediately frozen at $-20^{\circ} \mathrm{C}$ until analysis.

\section{Measurement of amiodarone and}

$\mathrm{N}$-desethyl-amiodarone concentrations

Amiodarone and its main metabolite $\mathrm{N}$-desethylamiodarone were quantified in plasma and fat tissue with a validated method, using liquid chromatography coupled to ion trap mass spectrometry detection (LC/MS/MS) with electrospray ionization interface. An enzymatic lipase solution was added to each tube containing fat tissue and incubated at $50^{\circ} \mathrm{C}$ for $16 \mathrm{~h}$. The extraction procedure for fat digest or plasma was carried out using diethyl ether. AM-25, a compound similar to rimonabant, was used as internal standard. Chromatography was performed on a $5-\mu m C_{18}$ column maintained at $30^{\circ} \mathrm{C}$. The elution was achieved isocratically with a mobile phase at a flow rate of $200 \mu \mathrm{l} \mathrm{min}{ }^{-1}$. Data were collected either in full-scan MS mode at $m / z \quad 100-650$ or in full-scan MS/MS mode, selecting the ion $\mathrm{m} / \mathrm{z} 646.0$ for amiodarone, $\mathrm{m} / \mathrm{z} 617.9$ for $\mathrm{N}$-desethyl-amiodarone, and $\mathrm{m} / \mathrm{z} 551.1$ for internal standard. The most intense daughter ion of amiodarone $(\mathrm{m} / \mathrm{z}$ 572.8), N-desethyl-amiodarone ( $m / z$ 546.8) and internal standard ( $\mathrm{m} / \mathrm{z}$ 472.8) were used for quantification.

Tissue digestion and extraction were validated using drug-free adipose tissue obtained from autopsy of deceased patients, which were spiked with both analytes. Calibration curves were linear for both compounds in plasma and fat tissue in the $0.1-5 \mathrm{mg} \mathrm{l}^{-1}$ and $1-100 \mathrm{mg} \mathrm{kg}^{-1}$ range, with limits of quantification being $0.1 \mathrm{mg} \mathrm{l}^{-1}$ and $1 \mathrm{mg} \mathrm{kg}^{-1}$, respectively. Accuracy and precision of the assay were measured by analysing 18 quality controls samples for 3 days. At the concentration of 0.15 and $4 \mathrm{mg} \mathrm{l}^{-1}$, intraday precisions were 6.5 and $5.0 \%$ for amiodarone and 7.7 and $7.3 \%$ for $\mathrm{N}$-desethyl-amiodarone, whereas interday precisions were 14.9 and $5.0 \%$ for amiodarone and 3.4 and $13.6 \%$ for $\mathrm{N}$-desethyl-amiodarone, respectively. Accuracies were in the $92.2-105.7 \%$ range.

\section{Other variables measured}

Age, gender, weight, creatinine, transaminases, prothrombin time, thyroid stimulating hormone, electrocardiogram, chest $\mathrm{x}$-ray, presence of respiratory or ocular symptoms, and any concomitant drug treatment were recorded. Any present or previous amiodarone-related adverse effect, defined as such by the patient's attending physicians, was recorded. We included amiodarone-related adverse effects diagnosed prior to sampling if the adverse effect persisted and the patient was still receiving amiodarone without any recent dose modification (last 3 months). History of amiodarone administration (loading dose(s), successive maintenance dose(s)) was retraced for every patient by examining all prescription records and hospital charts, and total time on treatment and cumulated dose were calculated. When analysing the relationship with the development of adverse effects, cumulated time and dose were adjusted to the moment when the adverse effect was first diagnosed. Patients were examined $24 \mathrm{~h}$ after the punctures to exclude local complications.

\section{Statistical analysis}

Reproducibility of measures in adipose tissue was tested using Bland-Altman plot. For all other analyses, the average of the two concentrations measured in adipose tissue was employed. Relationship between continuous variables was explored using linear and nonlinear regression models (the best fitting model was retained). It was estimated that 30 patients were needed to reach $80 \%$ probability of proving a significant correlation of $\geq 0.50$. Amiodarone-related toxicity was treated as a binary variable (any toxicity, none) and its potential associations examined by logistic regression. For all analyses, a twotailed $P$-value of $\leq 0.05$ was considered significant. All calculations were done using SAS software (SAS Institute Inc., Cary, NC, USA).

\section{Results}

From July 2006 to October 2007, 67 consecutive patients were evaluated and 30 patients included. Reasons for noninclusion were: impossible to retrace history of amiodarone administration $(n=19) ;<3$ months on amiodarone treatment $(n=10)$; unable to give informed consent $(n=4)$; refused to participate $(n=4)$. Subcutaneous adipose tissue aspiration was well tolerated: $73 \%$ of patients considered the pain equal to or lower than a standard venous puncture, and the only complication was local haematoma in two patients ( 2 and $3 \mathrm{~cm}$ diameter, respectively).

\section{Characteristics of included patients}

The characteristics of included patients are displayed in Table 1. Mean age was 75 years, and men predominated. The most frequent indication for amiodarone treatment was prevention of recurrences of atrial fibrillation. All patients were receiving low maintenance doses of amiodarone: 100-200 mg taken 5-7 days a week. Except for four patients with lone atrial fibrillation, all patients had diverse cardiac conditions, the most frequent being coronary disease, hypertensive heart disease and idiopathic dilated cardiomyopathy. Only one patient had prior hepatic failure, secondary to autoimmune chronic hepatitis. 


\section{Table 1}

Characteristics of included patients

\begin{tabular}{|c|c|}
\hline Male/female, no. & $19 / 11$ \\
\hline Age, mean \pm SD (range) years & $75 \pm 14(35-94)$ \\
\hline Body mass index, mean \pm SD (range) & $23.7 \pm 4.5(15-36)$ \\
\hline Creatinine clearance, mean $\pm \mathrm{SD}, \mathrm{ml} \mathrm{min}^{-1}$ & $60 \pm 25$ \\
\hline Patients with creatinine clearance $<50 \mathrm{ml} \mathrm{min}{ }^{-1}$, no. & 10 \\
\hline Patients with creatinine clearance $<30 \mathrm{ml} \mathrm{min}{ }^{-1}$, no. & 2 \\
\hline Patients with a diagnosis of hepatic failure, no. & 1 \\
\hline Coronary disease, no. & 10 \\
\hline Hypertensive heart disease, no. & 7 \\
\hline Non-ischaemic dilated cardiomyopathy, no. & 5 \\
\hline Other cardiac conditions (valvular disease, cor pulmonale), no. & 3 \\
\hline Chronic important noncardiac conditions, no. & 19 \\
\hline \multicolumn{2}{|l|}{ Amiodarone treatment } \\
\hline Total time of treatment, mean \pm SD (range), months & $36 \pm 43(3-148)$ \\
\hline Total cumulated dose, mean \pm SD (range), g & $205 \pm 263(15-968)$ \\
\hline \multicolumn{2}{|l|}{ Associated treatments } \\
\hline Digoxin, no. & 2 \\
\hline Diuretics, no. & 16 \\
\hline$\beta$-Blockers, no. & 9 \\
\hline Calcium-channel blockers, no. & $1^{*}$ \\
\hline Statins, no. & $7+$ \\
\hline
\end{tabular}

*The patient received amlodipine. †Four patients received pravastatin, two simvastatin and one atorvastatin. no., number of patients; SD, standard deviation.

\section{Relationships between plasma and adipose tissue concentrations}

Concentrations of amiodarone and N-desethylamiodarone in plasma were well correlated with the respective concentrations in adipose tissue (Figure $1 \mathrm{~A}, \mathrm{~B}$, Figure S1). Concentrations in fat tissue were largely superior to those in plasma for amiodarone (mean fat tissue: plasma ratio 55, range 4-226), but less so for $\mathrm{N}$-desethylamiodarone (mean fat tissue : plasma ratio 3 , range 0.12 11 ), reflecting the known fact that $\mathrm{N}$-desethyl-amiodarone is less lipophilic. The mean amiodarone: $\mathrm{N}$-desethylamiodarone ratio was 0.85 (range $0.6-1.5$ ) in plasma and 30 (range 5.2-107) in adipose tissue. No significant correlation appeared between concentrations in fat tissue and body weight or body mass index.

Amiodarone concentrations in fat tissue measured at different sampling points (abdominal wall and lumbar area) were well correlated (Figure 1C). When studied in a Bland-Altman plot, the limits of agreement were large (variations of -66 to $57 \mathrm{mg} \mathrm{kg}^{-1}$ in measures, Figure 1D). This high variability appeared to be generated mainly by a few unusually discordant measures. Contamination of fat tissue samples with blood during the aspiration, which was observed in some cases, was suspected to be the cause.

\section{Correlation of concentrations with doses and time}

Plasma concentrations of amiodarone were significantly correlated with daily maintenance doses, especially when adjusted by body weight (Figure 2A). In contrast, adipose tissue concentrations showed no clear correlation with daily doses (Figure $2 \mathrm{C}$ ).

Concerning cumulated doses, no significant correlation was seen between amiodarone concentrations, either in plasma or in fat tissue, and total administered dose or total treatment duration (Figure 2B,D), even when cumulated dose was adjusted by body weight or body mass index.

Levels of N-desethyl-amiodarone followed exactly the same correlation (or lack of it) as amiodarone with respect to maintenance dose, cumulated dose or cumulated time (Figure S2).

\section{Amiodarone-related adverse effects and associated risk factors}

At the time of patient enrolment and sample collection, 11 patients had developed adverse effects requiring changes in amiodarone treatment (dose reduction or withdrawal) and/or additional specific treatment: eight patients had hypothyroidism needing L-thyroxin supplementation (three of whom also had symptomatic bradycardia or 

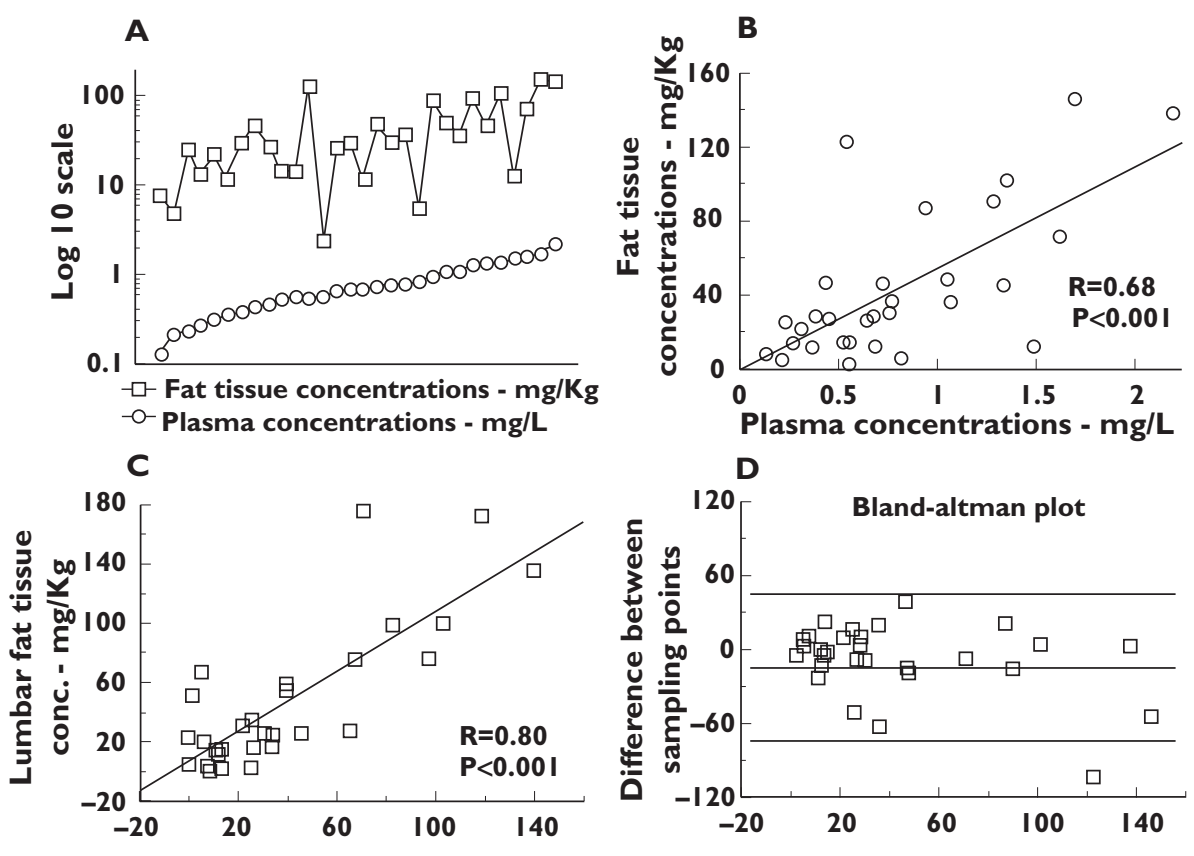

Abdominal fat tissue conc. - $\mathrm{mg} / \mathrm{Kg}$

Average of the 2 fat tissue sampling points

\section{Figure 1}

Amiodarone concentrations in plasma and adipose tissue. Conc., concentrations. (a) Concentrations of amiodarone in adipose tissue and in plasma, in logarithmic scale, with observations ordered by plasma concentrations. (b) Linear regression of concentrations obtained in fat tissue with regard to concentrations in plasma. (c) Linear regression of concentrations obtained in the two simultaneous samples of adipose tissue performed. (d) Bland-Altman plot displaying the difference between measures at the two different sampling points of adipose tissue against its average, in mg $\mathrm{kg}^{-1}$. The limits of agreement are defined by the mean difference (medium line) + - - twice the standard deviation of the differences (top and bottom lines)

advanced heart block requiring pace-maker implantation); two patients presented with pulmonary toxicity (one diagnosed by bronchoalveolar lavage, the other by clinical criteria and exclusion of other aetiologies, both improved after amiodarone withdrawal); and one patient had amiodarone-related severe subacute hepatitis (Table S1).

After adjusting to the moment that amiodaronerelated adverse effects were first diagnosed, the incidence of any adverse effect was significantly associated with the cumulated time of treatment (time to development of the adverse effect) and the cumulated dose received (Table 2 and Table S1). Nine of the 12 patients who had received amiodarone for $\geq 2$ years - equivalent to a cumulated dose of $\geq 145 \mathrm{~g}$ - developed adverse effects, including the two patients with pulmonary toxicity, compared with only two of 18 patients with lower treatment duration or cumulated dose [relative risk $6.75 ; 95 \%$ confidence interval $(\mathrm{Cl}) 1.8,26)$.

Conversely, amiodarone toxicity was not associated with plasma or fat tissue concentrations, or with daily maintenance dose (Table 2). Multivariate analysis (stepwise logistic regression) including amiodarone concentrations in plasma and fat, cumulated and daily doses, and treatment time, yielded the same results: only the cumulated time or the cumulated dose (these two variables being directly linked) appeared significantly associated with the incidence of amiodarone toxicity.

A specific analysis of hypothyroidism - as it was the most frequent adverse effect (eight of 11 patients) yielded the same results: its occurrence was significantly associated with the duration of treatment [odds ratio (OR) per year of treatment $1.34,95 \% \mathrm{Cl} 1.04,1.74 ; P=0.025]$ and cumulated dose (OR per $\mathrm{g} \mathrm{kg}^{-1}$ body weight $1.27,95 \% \mathrm{Cl}$ $1.01,1.6 ; P=0.04$ ), but not with maintenance dose or with plasma or fat tissue concentrations of amiodarone or $\mathrm{N}$-desethyl-amiodarone.

\section{Discussion}

In this work, we have studied the relationships between administered doses of amiodarone, its concentrations in plasma and in fat tissue, and the incidence of related adverse effects, in a series of consecutive patients on chronic therapy with this drug. We found a direct and significant correlation between daily maintenance dose and plasma concentrations, and between concentrations in plasma and in adipose tissue. Contrary to what we expected, no significant correlation was found between adipose tissue concentrations and cumulated doses or 

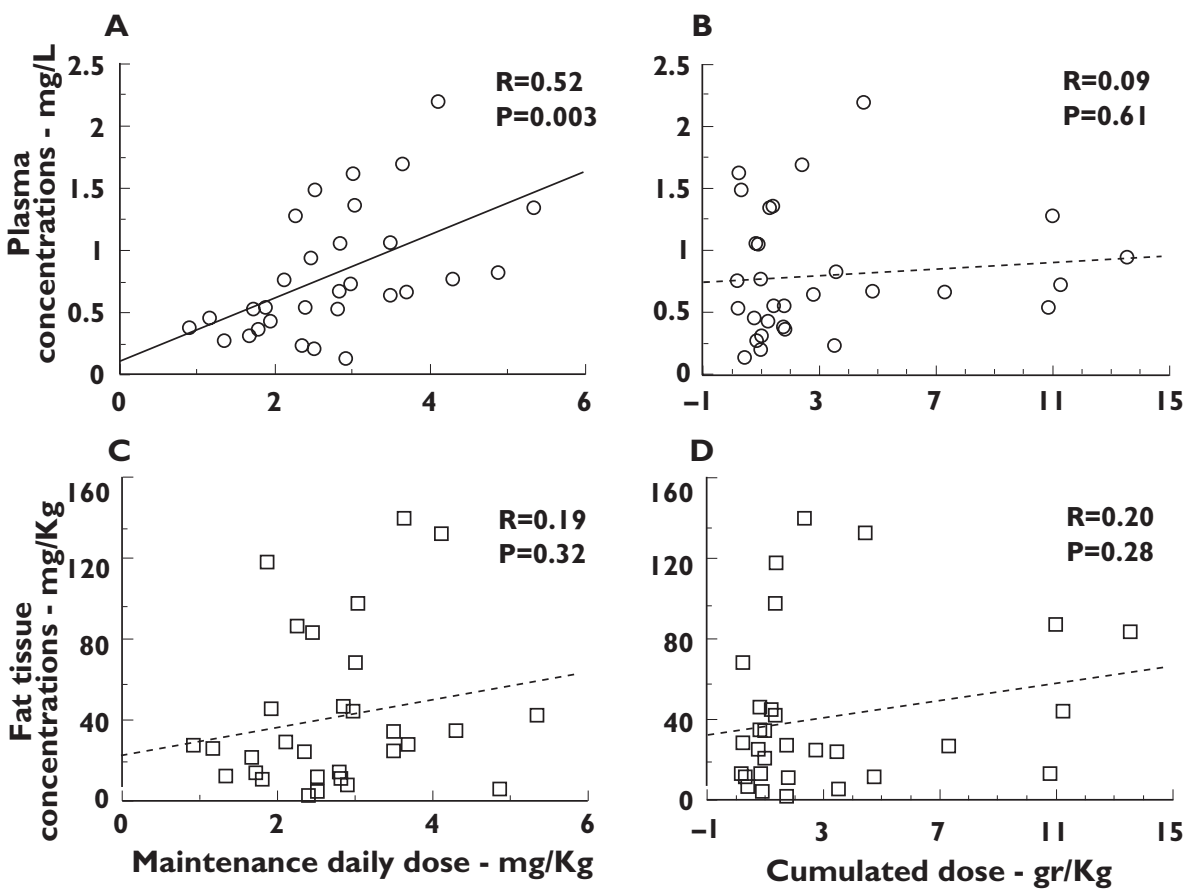

\section{Figure 2}

Relationships of amiodarone concentrations with cumulated and daily doses. Conc., concentrations. Correlations between amiodarone concentrations, in plasma and fat tissue, and maintenance and cumulated doses, adjusted by patient body weight, in Kg. Linear regression is displayed for all four analyses; nonlinear models were also explored but did not fit better

Table 2

Risk factors for amiodarone toxicity

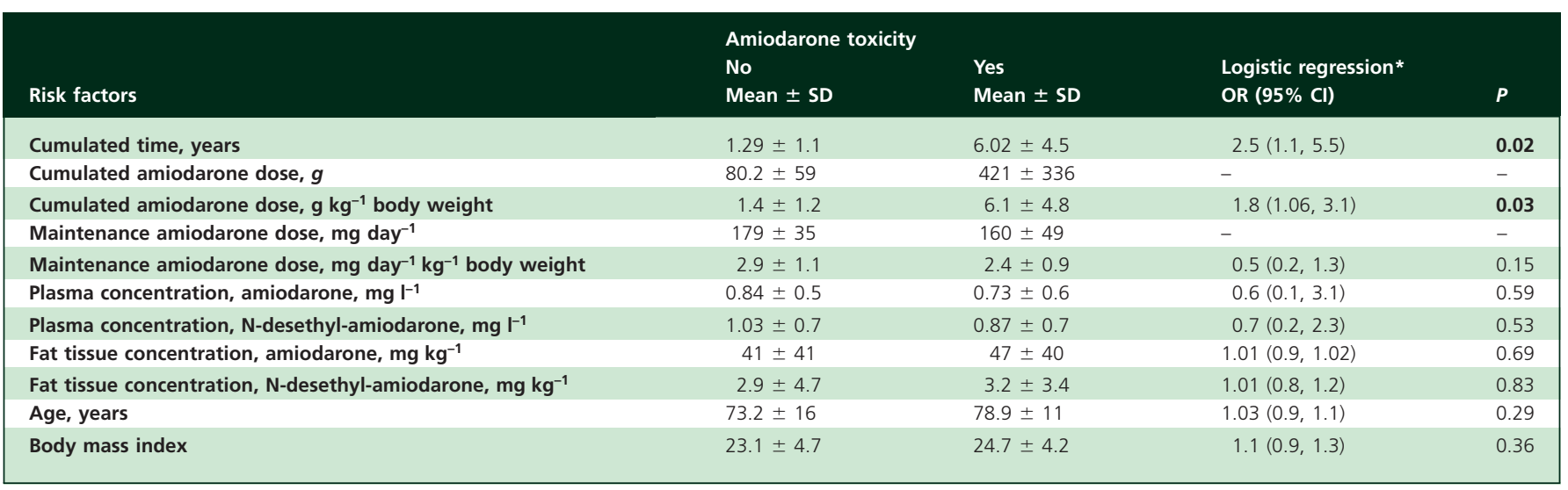

*Univariate analysis, OR given for each increase in 1 unit of the variable analysed. $95 \% \mathrm{Cl}, 95 \%$ confidence interval; OR, odds ratio; SD, standard deviation.

total time of treatment. Finally, we recorded a high frequency of amiodarone-related adverse effects, predominantly hypothyroidism, which were strongly associated with the total duration of amiodarone treatment and, consequently, with the cumulated dose. However, adverse effects were not related to amiodarone or N-desethylamiodarone concentrations, either in plasma or in fat tissue.
We had hypothesized that an excessive accumulation of amiodarone would occur in adipose tissue with increased treatment duration, as suggested by some studies $[14,16,17]$ and modelled by some authors [10]. The increasing tissue concentrations would have provided a good explanation for the rising incidence of amiodaronerelated toxicity over time. This could have provided a rationale for the reduction of maintenance doses, which has 
already been explored in some pilot studies [19, 20], as a means to limit such chronic accumulation in tissues. However, the results we obtained do not support this hypothesis. Amiodarone concentrations in fat tissue were much higher than in plasma, as expected for a very lipophilic drug, but without any significant correlation with treatment duration or with cumulated dose. Many patients who had been treated for only a few months reached concentrations in fat tissue equal or superior to those of other patients treated for several years.

Instead, our findings are better explained supposing that amiodarone follows a typical two-compartment (plasma and tissue) pharmacokinetic model, where plasma concentrations depend on repeated maintenance doses and concentrations in tissues are linked to those obtained in plasma. This model implies that a steady state is reached in each compartment and kept stable thereafter, without further increases in concentrations. This was also the best fitting model found by Pollak et al., based on repeated measurements of serum levels, in the largest population study conducted with amiodarone to date [12].

Surprisingly, we found no direct correlation between concentrations in fat tissue and maintenance daily doses. This could in part reflect the relatively poor reproducibility observed in the assay of amiodarone in fat tissue. However, in that case we would have expected that the correlation found between fat tissue concentrations and plasma concentrations were affected also. Otherwise, interaction with other drugs may have altered amiodarone pharmacokinetics in some patients, either modifying its metabolism, CYP3A4 mediated, or its absorption and elimination, mediated by the adenosine triphosphate-dependent efflux transporter P-glycoprotein. For example, one patient received amlodipine, which is partially metabolized by CYP3A4, and seven patients received statins, which can inhibit CYP3A4, although in four of them the drug was pravastatin, much less metabolized by CYP3A4. Calcium channel blockers also interfere with P-glycoprotein, as well as digoxin, which some other patients were receiving. Finally, if not caused by those external factors, the lack of correlation between concentrations in fat tissue and maintenance dose would indicate that interindividual differences in adipose tissue proportion and distribution do not parallel interindividual variability in plasma concentrations, suggesting that adipose tissue might not accumulate amiodarone and its metabolites as other tissues.

Our results concerning amiodarone-related adverse effects, which in our study concern mainly hypothyroidism, are more difficult to interpret. They show a clear association between the risk of developing hypothyroidism, or any adverse effect, and the exposure time to amiodarone and thus, with cumulated dose, a collinear variable. In contrast, no association was found with concentrations, either in plasma or in fat tissue. Other studies have yielded conflicting results. Two studies $[17,21]$ found significantly higher serum levels of amiodarone and its metabolite in patients with adverse effects. One of these two studies, by Gonska et al. [17], also found higher concentrations in subcutaneous adipose tissue in patients with adverse effects. Other studies have found only a weak correlation [22, 23], or no association $[12,24]$ of serum amiodarone levels with toxicity. Shukla et al. [13] found, as we did, that adverse events, mainly cutaneous and thyroid, were more likely to occur with increasing duration of treatment and cumulated dose. Cumulated time and dose were not assessed in the other mentioned studies. Our results and those of Shukla suggest that, in long-term treatment, constant and prolonged exposure to amiodarone could be a stronger risk factor for adverse effects than the absolute concentrations reached in plasma and tissues. Another possibility is that the drug is distributed differently to sites of toxicity than to adipose tissue.

Possible limitations of our study are: (i) the relatively small number of patients included, which was calculated to provide enough power to detect a correlation between doses and concentrations and not to assess the relation between amiodarone concentrations and adverse events; (ii) measurements were performed only once in each patient, so intraindividual variability in time could not be assessed; (iii) the incidence of amiodarone-related adverse effects was probably overestimated in this series of patients, since all were recruited in a university hospital, a referral centre; (iv) variability of the measured concentrations of amiodarone might have been increased by interaction with other drugs or poor reproducibility of the assay, as discussed above; and (v) the impossibility of measuring amiodarone concentrations in the tissues affected by adverse effects (thyroid, lung, liver), so abnormal accumulation or sequestration of amiodarone specific to those tissues can not be excluded.

In conclusion, observed concentrations of amiodarone and $\mathrm{N}$-desethyl-amiodarone in plasma and subcutaneous fat tissue in patients on long-term treatment are better related to maintenance daily dose than to cumulated dose or treatment duration. Amiodarone does not appear to accumulate in excess in fat tissue in patients treated for $>3$ months. Clinically relevant adverse effects of amiodarone, for the most part hypothyroidism, were significantly associated with longer duration of treatment and larger cumulated doses. More than 2 years on amiodarone, or $>145 \mathrm{~g}$ of cumulated dose, clearly increased the risk of related toxicity in this series. Conversely, hypothyroidism and adverse events in general were not correlated with higher amiodarone concentrations, whether in plasma or in adipose subcutaneous tissue. Therefore, the measurement of amiodarone and $\mathrm{N}$-desethyl-amiodarone in plasma or subcutaneous fat tissue does not seem useful in accurate prediction of the risk of developing adverse effects in patients on chronic treatment with low-dose amiodarone. Further research, involving a larger number of patients and stratifying them according to the type of adverse effect, 
would be needed to better quantify the increased incidence of adverse effects over time associated with longterm amiodarone therapy.

\section{Competing interests}

None to declare.

This work was supported by the Unité de Recherches Thérapeutiques, Hôpital Lariboisière, Paris. The sponsor had no role in the conduct of the study, or in the collection, analysis, and interpretation of the data. It had no role regarding the preparation, review and approval of the manuscript, or the decision to publish. Dr Florence Beauvais and M. Marc Etchegoin helped to recruit patients. Dr Guy Simoneau handled samples stock and transport. Ms Pierrette Fouquet assisted in all the administrative tasks.

\section{REFERENCES}

1 Vassallo P, Trohman RG. Prescribing amiodarone: an evidence-based review of clinical indications. JAMA 2007; 298: 1312-22.

2 Lafuente-Lafuente C, Mouly S, Longas-Tejero M, Bergmann J. Antiarrhythmics for maintaining sinus rhythm after cardioversion of atrial fibrillation. Cochrane Database Syst Rev 2007; 4: CD005049.

3 Vorperian VR, Havighurst TC, Miller S, January CT. Adverse effects of low dose amiodarone: a meta-analysis. J Am Coll Cardiol 1997; 30: 791-8.

4 Amiodarone Trials Meta-Analysis Investigators. Effect of prophylactic amiodarone on mortality after acute myocardial infarction and in congestive heart failure: meta-analysis of individual data from 6500 patients in randomised trials. Lancet 1997; 350: 1417-24.

5 Harris L, McKenna WJ, Rowland E, Holt DW, Storey GC, Krikler DM. Side effects of long-term amiodarone therapy. Circulation 1983; 67: 45-51.

6 Batcher EL, Tang XC, Singh BN, Singh SN, Reda DJ, Hershman JM; SAFE-T Investigators. Thyroid function abnormalities during amiodarone therapy for persistent atrial fibrillation. Am J Med 2007; 120: 880-5.

7 Dusman RE, Stanton MS, Miles WM, Klein LS, Zipes DP, Fineberg NS, Heger JJ. Clinical features of amiodarone-induced pulmonary toxicity. Circulation 1990; 82: 51-9.

8 Adams PC, Holt DW, Storey GC, Morley AR, Callaghan J, Campbell RW. Amiodarone and its desethyl metabolite: tissue distribution and morphologic changes during long-term therapy. Circulation 1985; 72: 1064-75.

9 Tedelind S, Larsson F, Johanson C, van Beeren HC, Wiersinga WM, Nyström E, Nilsson M. Amiodarone inhibits thyroidal iodide transport in vitro by a cyclic adenosine 5'-monophosphate- and iodine-independent mechanism. Endocrinology 2006; 147: 2936-43.

10 Weiss $M$. The anomalous pharmacokinetics of amiodarone explained by nonexponential tissue trapping. J Pharmacokinet Biopharm 1999; 27: 383-96.

11 Holt DW, Tucker GT, Jackson PR, McKenna WJ. Amiodarone pharmacokinetics. Br J Clin Pract Suppl 1986; 44: 109-14.

12 Pollak PT, Bouillon T, Shafer SL. Population pharmacokinetics of long-term oral amiodarone therapy. Clin Pharmacol Ther 2000; 67: 642-52.

13 Shukla R, Jowett NI, Thompson DR, Pohl JE. Side effects with amiodarone therapy. Postgrad Med J 1994; 70: 492-8.

14 Candinas R, Frielingsdorf J, Ha HR, Carrel T, Turina M, Follath F. Myocardial amiodarone concentrations after shortand long-term treatment in patients with end-stage heart failure. Eur J Clin Pharmacol 1998; 53: 331-6.

15 Giardina EG, Schneider M, Barr ML. Myocardial amiodarone and desethylamiodarone concentrations in patients undergoing cardiac transplantation. J Am Coll Cardiol 1990; 16: 943-7.

16 Barbieri E, Conti F, Zampieri P, Trevi GP, Zardini P, d'Aranno V, Latini R. Amiodarone and desethylamiodarone distribution in the atrium and adipose tissue of patients undergoing short- and long-term treatment with amiodarone. J Am Coll Cardiol 1986; 8: 210-3.

17 Gonska BD, Bethge KP, Wagner H, Bosse K, Köbberling J, Quentin CD, Kreuzer H. Amiodarontherapie - Verhalten von Serum- und Fettgewebskonzentrationen [Amiodarone therapy - behavior of serum and fatty tissue concentrations]. Klin Wochenschr 1986; 64: 219-26.

18 Beynen AC, Katan MB. Rapid sampling and long-term storage of subcutaneous adipose-tissue biopsies for determination of fatty acid composition. Am J Clin Nutr 1985; 42: 317-22.

19 Mahmarian JJ, Smart FW, Moyé LA, Young JB, Francis MJ, Kingry $\mathrm{CL}$, Verani MS, Pratt CM. Exploring the minimal dose of amiodarone with antiarrhythmic and hemodynamic activity. Am J Cardiol 1994; 74: 681-6.

20 Jong GP, Chang MH, Chang TC, Chou P, Fu CY, Tien LY, Chen CY, Ma TC. Long-term efficacy and safety of very-low-dose amiodarone treatment for the maintenance of sinus rhythm in patients with chronic atrial fibrillation after successful direct-current cardioversion. Chin Med J (Engl) 2006; 119: 2030-5.

21 Rotmensch HH, Belhassen B, Swanson BN, Shoshani D, Spielman SR, Greenspon AJ, Greenspan AM, Vlasses PH, Horowitz LN. Steady-state serum amiodarone concentrations: relationships with antiarrhythmic efficacy and toxicity. Ann Intern Med 1984; 101:462-9.

22 Raeder EA, Podrid PJ, Lown B. Side effects and complications of amiodarone therapy. Am Heart J 1985; 109: 975-83.

23 Falik R, Flores BT, Shaw L, Gibson GA, Josephson ME, Marchlinski FE. Relationship of steady-state serum concentrations of amiodarone and desethylamiodarone to therapeutic efficacy and adverse effects. Am J Med 1987; 82: $1102-8$ 
Amiodarone concentrations in fat tissue and toxicity $\mathrm{B}] \mathrm{CP}$

24 Paling TJ, Siebers RW, Burgess CD, Taylor C, Purdie G. Individual variability of amiodarone distribution in plasma and erythrocytes: implications for therapeutic monitoring. The Drug Monit 1989; 11: 121-6.

Supporting Information

Additional Supporting Information may be found in the online version of this article:

Figure S1

$\mathrm{N}$-desethyl-amiodarone concentrations in plasma and adipose tissue. Conc., concentrations. (a) Concentrations of $\mathrm{N}$-desethyl-amiodarone in adipose tissue and in plasma, with observations ordered by plasma concentration. (b) Linear regression of concentrations obtained in fat tissue with regard to concentrations in plasma. (c) Linear regression of concentrations obtained in the two simultaneous samples of adipose tissue performed. (d) Bland-Altman plot displaying the difference between measures at the two sampling points of adipose tissue against its average, in $\mathrm{mg} \mathrm{kg}^{-1}$. The limits of agreement are defined by the mean difference (medium line) +/- twice the standard deviation of the differences (top and bottom lines)

Figure S2

Relationships of $\mathrm{N}$-desethyl-amiodarone concentrations with cumulated and daily doses. Conc., concentrations. Correlations between $\mathrm{N}$-desethyl-amiodarone concentrations, in plasma and fat tissue, and maintenance and cumulated doses of amiodarone, adjusted by patient body weight, in $\mathrm{kg}$. Linear regression is displayed for all four analyses; nonlinear models were also explored but did not fit better Table S1

Detail of amiodarone adverse effects, cumulated time, doses and concentrations

Please note: Wiley-Blackwell are not responsible for the content or functionality of any supporting materials supplied by the authors. Any queries (other than missing material) should be directed to the corresponding author for the article.

Br J Chin Pharmacol / $\mathbf{6 7 : 5 ~ / ~} 519$ 